\title{
Maximum Impact Flow for optics teaching
}

\author{
J. McCarthy, R. A. Moore
}

J. McCarthy, R. A. Moore, "Maximum Impact Flow for optics teaching," Proc. SPIE 9664, Ninth International Topical Meeting on Education and Training in Optics and Photonics, 966408 (24 October 2005); doi: 10.1117/12.2207528

SPIE Event: Ninth International Topical Meeting on Education and Training in Optics and Photonics, 2005, Marseille, France 
Ref ETOP003

\title{
Maximum Impact Flow for Optics Teaching
}

\author{
J. McCarthy*1, R.A. Moore ${ }^{1}$ \\ ${ }^{1}$ Department of Electronic and Electrical Engineering, University of Dublin, \\ Trinity College, Dublin, Ireland
}

\begin{abstract}
Optics as a teaching tool is investigated within this paper, with special emphasis on the proposed use of a Maximum Impact Flow (MIF) in order to stimulate student's interests. MIF introduces a template in terms of individual steps and linked functionality. MIF is shown to fuse separate learning tools together into a cohesive unit. Prioritisation and teaching structures are also discussed with the promotion of technology identified as a
\end{abstract}

Teaching, MIF, Template, Structures.

\section{Key Words}

\section{Summary}

Introduction

From comic book superheroes to state of the art technology, optics and its influences can be seen worldwide. Both the teacher and educator need to constantly re-evaluate teaching tools in order to focus the student on the amazing capabilities and versatility of optical design. The increased sense of awareness and interest in this ever expanding area is a challenge that faces us all. Increased stage agency co-operation, use of MIF (Maximum Impact Flow), and a development in the road map of education that includes a strong scientific theme are challenges that need to be addressed sooner rather than later.

The development of the optics industry continues to grow at a staggering pace1,2 In order to address this ever increasing market, it is necessary to have scientists in place that can play a proactive role in meeting the challenges of the future. This paper outlines the importance of education as well as the adoption of a fixed template in a learning environment

\section{Promotion of Technology}

The importance of promotion in the photonics industry is vital from both a marketing and educational perspective. Millions of dollars are spent annually by leading companies and in the U.S. alone, spending in the fiber optics market has more than tripled this decade from $\$ 4.1$ billion in 1990 to $\$ 14.6$ billion in 19993 .

However, little or no investment is afforded to the nurturing and fostering of physics at the school level. In an era when technology companies are bemoaning the lack of suitable scientists, it is noteworthy that these same companies fail to see the link between the student of today and the scientists of tomorrow. It has been shown that 
even the most simple of experiments can stimulate the student into taking an interest into the basics of photonics4.

Government agencies too, have shown an increasing awareness in this area and many state funded activities are now in place. However, it is clear that without the help, both in terms of finance and ideas, then this will not be enough.

It has been shown5 that in an average day there will be countless numbers of encounters with optical technology. Transferring this real scenario into an educational tool is a challenge that rests not just with the teacher but all of us. Major advances in the optics industry are ready to take place6, but realizing this vision will take a reordering of research priorities, better coordination among agencies and industries engaged in optical science, and government leadership in focusing the efforts of the research community.

\section{Experiments}

An optical demonstration transforms what could be seen in the classroom as dull ideas into a vibrant display that can stir the imagination of young minds. Laser shows have been proven as a vital tool in the education of young student7. Programmes such as HOO8, allow for development of scientific elements across a broad spectrum of cultures and environments. Simple experiments are an ideal way to alert the classroom to the presence of photonics in the modern world9.Reflection, Refraction, and diffraction offer simple yet effective ways of introducing the ideas of optics into the classroom. These experiments can be made hands on in order to maximise their impact in the classroom.

One off experiments, while momentarily effective, lacks the necessary momentum to influence a student's learning curve. Therefore, experiments and real life situations from photonics must be adapted for the student's continuous academic development. Career choice is dependant on a variety of factors, or layers, such as environment, family situation, natural inclinations and outside influences, to name but a few. The use of a Maximum Impact Flow (MIF) allows a defined template to be adopted in the promotion and education of optics throughout the student's life. Many agencies and courses are short-term results driven without puncturing their views with long term development prospects. Adoption of MIF allows one to define the steps necessary in order to provide future throughput in the areas of optics, and indeed science in general.

\section{Maximum Impact Flow:}

Identification of scientific elements (optics) within the student's environments, i.e. CD player, shopping checkout etc

- Fostering this with a series of interactive demonstrations

- Development of this awareness with a series of online programmes

- Cross-Fertilisation - i.e. an optics day at a National Park to re-enforce point 1. IDENTIFICATION The task of identification is an important step in the proposed flow. It is used as a gateway to the other steps and therefore time and effort should be dedicated to delivering templates of identification within which the educator (parent and teacher) can work. 


\section{FOSTERING}

Showcase demonstrations, as well as hands on-experiments develop the theme of education through interaction. Linking visual and tactile approaches into the MIF provides a strong foundation for subsequent development.

\section{DEVELOPMENT}

With the continued expansion in the use of the world-wide web, it is of fundamental importance that strategies are adopted inline with this medium. Online teaching and demonstrations are suitable for class development as well as the utilisation of technologies that are otherwise unavailable to the Teacher.

\section{CROSS FERTILISATION}

Cross fertilisation allows the combination of diverse areas of interest, such as history, nature and science, into an effective common learning tool. Education through appreciation of our natural surroundings, such as diffraction of light in lakes etc, has maximum benefit from both an environmental and scientific strand. The adoption of techniques from outside the close confines of the scientific world, allows a greater access to the student's mind.

\section{Conclusions}

Each stage within this flow has already been identified as vital for the creation of the next generation scientists and engineers. The combination of these distinct points into a fluid and useable template gives a structure to this development. Education is essential to development, and in particular to what we today call "sustainable development." Sustainable development implies a balance between meeting immediate needs and looking to the future with a long-term vision. MIF fuses separate learning tools together into a cohesive unit. Long term results will not be visible for many years, but it is felt that the template suggested offers a benchmark that can be adopted by parent and educator alike for the benefit of science.

References
1. Technology Industry Growth Forecaster
http://www.photonics.org.uk/FibreOpticsHTML/fo-01-02.html

2000 MultiMedia Telecommunications Market Review and Forecast a joint publication produced by the Telecommunications Industry Association (TIA) and the MultiMedia Telecommunications Association (MMTA)

4. http://oemagazine.com/fromTheMagazine/mar01/edletter.html Harnessing Light is printed with permission by National Academy Press The Research Council's Committee on Optical Science and Engineering report "Harnessing Light: Optical Science and Engineering for the 21st Century"

Andrew Sabartnam, Charles Symons, Laser Entertainment and Light Shows in Education 7th International Conference on Education and Training in Optics and Photonics 2001 at Singapore (27 - 30 November 2001)

8. http://oemagazine.com/fromTheMagazine/current/spieworld_membernews.html

9. Rochester Regional Photonics Cluster, http://www.rrpc-ny.org/links.asp. 